\title{
20. MASS LOSS FROM ERUPTIVE STARS
}

\author{
Introductory Report
}

(Tuesday, September 16, 1969)

\author{
A. A. BOYARCHUK \\ Krymskaya Astrofizicheskaya Observatoriya, U.S.S.R.
}

\section{Methods of Determination of the Mass Loss Rate}

The previous reports by Lüst and by Pottasch concerned quasi-stationary mass loss. The loss is called quasi-stationary because the basic characteristics of a star, its temperature and its radius, do not change significantly during the time that a particle needs to flow from the stellar surface out to a point where the stellar influence is negligible. However there are many stars which show significant changes of temperature and radius within a short time. Very often these changes are accompanied by instantaneous ejection of a great amount of material. For example, during the outburst of a nova the ejected mass can reach $10^{-4} M_{\odot}$. Such effects are very appreciable in the so-called 'eruptive variables', in which the brightness changes spasmodically. It is very likely that the rate and the mechanism of mass loss of eruptive variables are different from that of normal stars.

I would like to say a few words on the determination of the mass loss rate. The mass loss rate is found mainly from three kinds of observations:

(i) Direct observation of nebulae, which are formed by the ejected material.

(ii) Investigation of line profiles in stellar spectra.

(iii) Observations of changes in the periods of binary systems.

The first kind gives the most reliable data about the mass-loss rate. One can derive the mass $M$ of an optically thin nebula from the total energy $E_{\mathrm{H} \beta}$ radiated at $\mathrm{H} \beta$. The mass of the nebula is given by

$$
M=V n_{e} m_{H},
$$

where $V$ is the volume of the radiating material. Similarly we have for the total emission

$$
E_{\mathrm{H} \beta}=V n_{e}^{2} \varepsilon_{\mathrm{H} \beta}\left(T_{e}\right),
$$

where $\varepsilon_{\mathrm{H} \beta}$ is the volume emissivity $\left(\mathrm{erg} \mathrm{cm}^{-3}\right.$ ) calculated for $n_{e}=1 \mathrm{~cm}^{-3}$. Eliminating $n_{e}$ between Equations (1) and (2) we obtain

$$
M=m_{\mathrm{H}} /\left(\frac{E_{\mathrm{H} \beta} V}{\varepsilon_{\mathrm{H} \beta}}\right) .
$$

From observations we obtain both the $\mathrm{H} \beta$ flux at the Earth (which yields $E_{\mathrm{H} \beta}$ ) and the angular size of the nebula (which gives $V$ ). Since $E_{\mathrm{H} \beta}$ is proportional to $R^{2}$ and $V$ to $R^{3}$, it is clear that the mass of the nebula is proportional to $R^{5 / 2}$, where $R$ is the distance to the nebula. Thus the main error in the value of the mass of the nebula 
arises from the uncertainty in the distance to the nebula. In addition the assumptions of optical thinness and of homogeneity of the nebula may not always be correct and may introduce errors.

The second method of determining the mass of the ejected material is based on line profiles. It is well known that emission lines with an absorption component at the short wavelength side are formed in an expanding gaseous envelope surrounding the star. The absorption component is formed in that part of the envelope which is projected on the stellar disc while the rest of the envelope gives the emission component. The mass loss rate is equal to

$$
\mathrm{d} M / \mathrm{d} t=\varrho V_{e j} 4 \pi R_{\mathrm{env}}^{2}
$$

where $\varrho$ and $R_{\text {env }}$ are the density and the radius of the envelope and $V_{e j}$ is the expansion velocity. All three values in the right-hand part of Equation (4) can be obtained from the observations. Kuhi (1964) developed a detailed theory of the line formation in an expanding envelope, which allows one to get $\varrho, V_{e j}$, and $R_{\text {env }}$ from the observed profile. Rough estimates of these values can be obtained from a visual inspection of spectrograms. The displacement of the absorption component relative to the emission component gives the expansion velocity. The relative intensities of the lines that are sensitive to dilution allow the determination of the radius of the envelope. A set of quality estimates as to the presence or absence of forbidden lines, the assumption that the density varies as $R^{-2}$ and so on, can give some information about the value of the density. Therefore the error in the density may reach several orders of magnitude if one does not undertake a detailed investigation. The stratification of radiation may bring in an additional difficulty. Finally, one needs to be certain that the material of the envelope leaves the star completely and does not return to the stellar surface in an invisible form. As a whole this second method gives less accurate results than the first.

The third method is based on the consideration of a change of the period of binary systems. Huang (1956) has shown that mass loss leads to an increase of the period, while mass exchange between the components leads to a decrease. Under simplifying assumptions Huang derived the following equation, which connects the rate of the mass loss from the primary component $\Delta m_{1}$ with a period change $\Delta p$

$$
\Delta p / p=2 \Delta m_{1} /\left(m_{1}+m_{2}\right)
$$

It is difficult to estimate the errors introduced by the simplifying assumptions. Also it is necessary to observe the period of binary systems with high precision in order to discover its change.

Now we will consider separately different types of eruptive stars: novae, symbiotic stars, and stars of the T Tauri type, the U Geminorum type and the UV Ceti type.

\section{Novae}

Novae are the uncontestable examples of objects which have significant rates of mass 


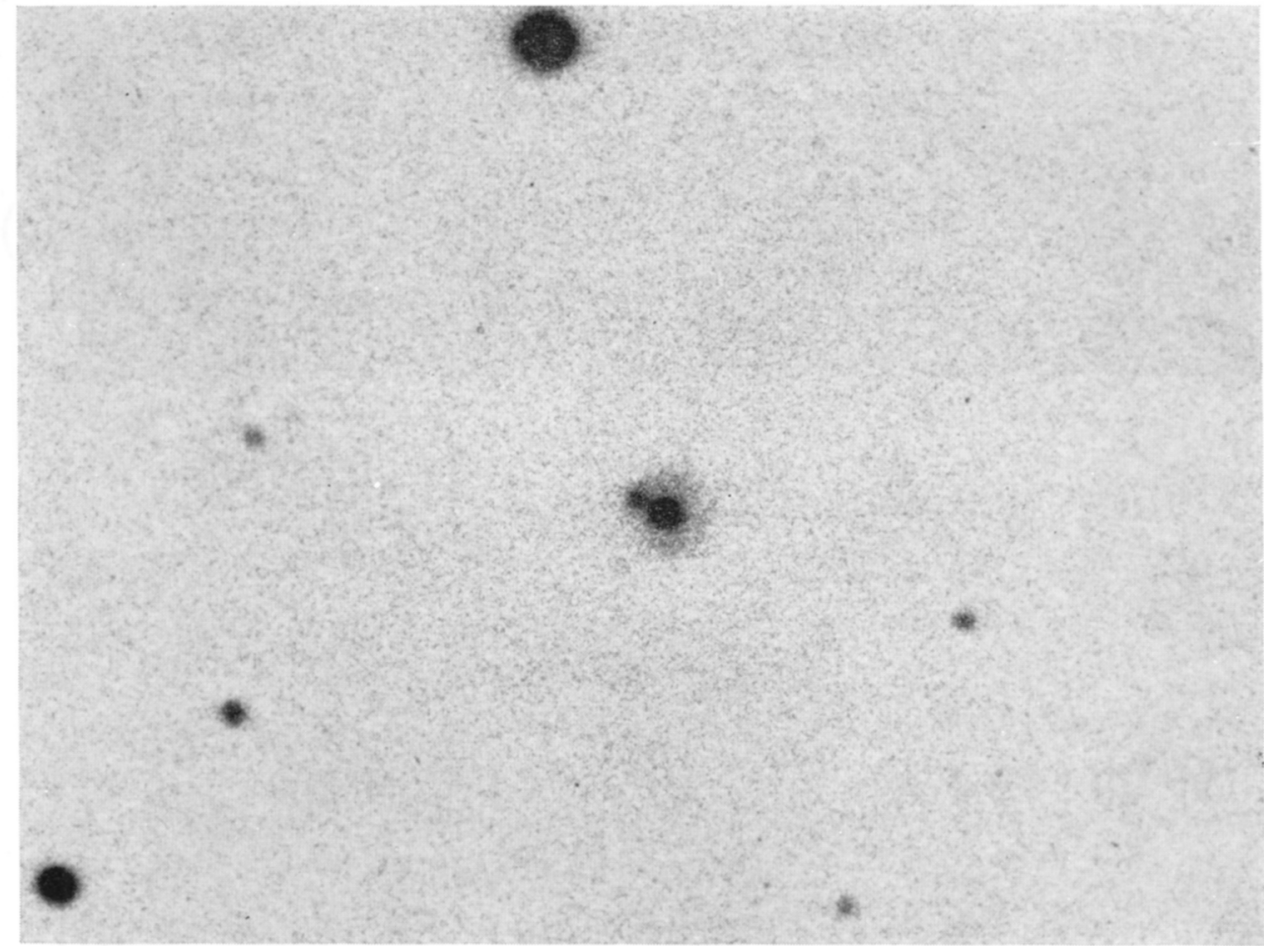

(a)

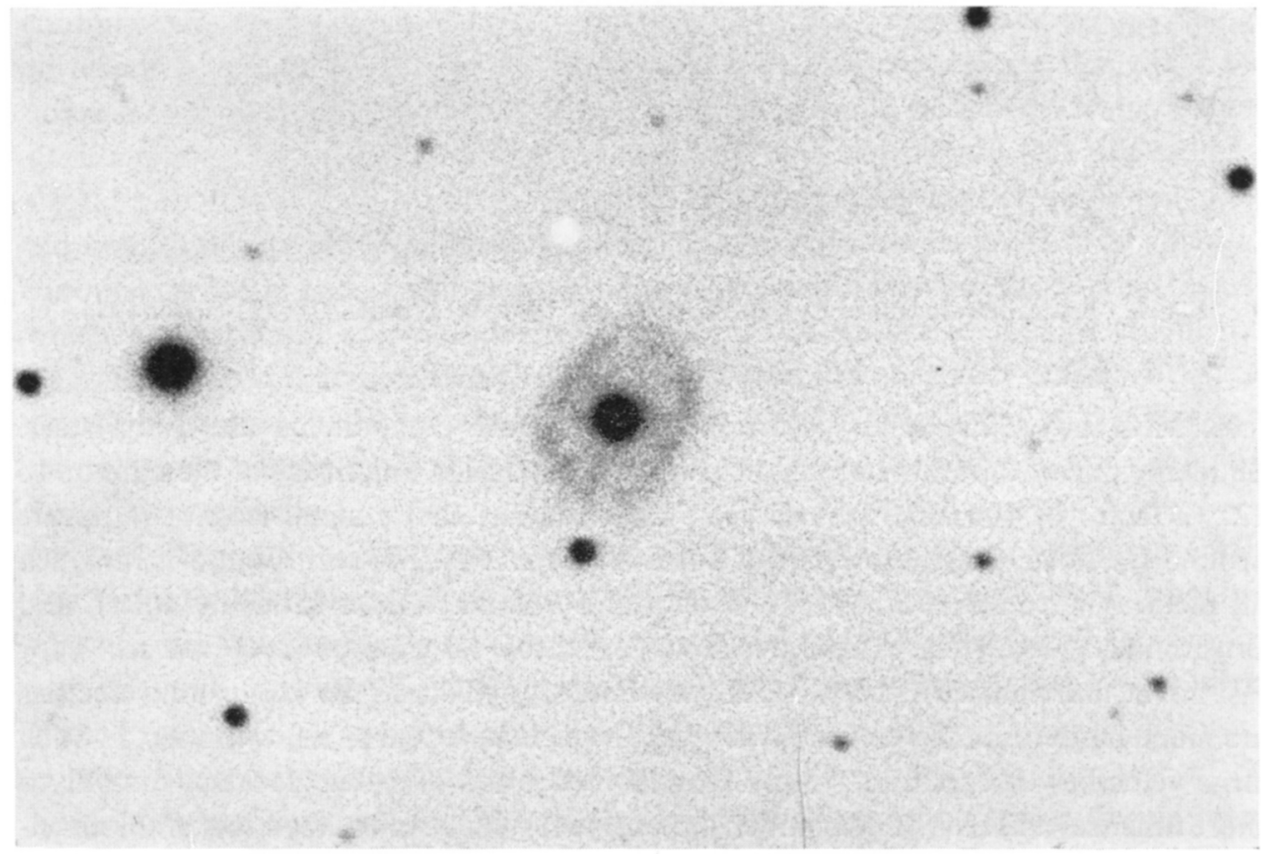

(b) 


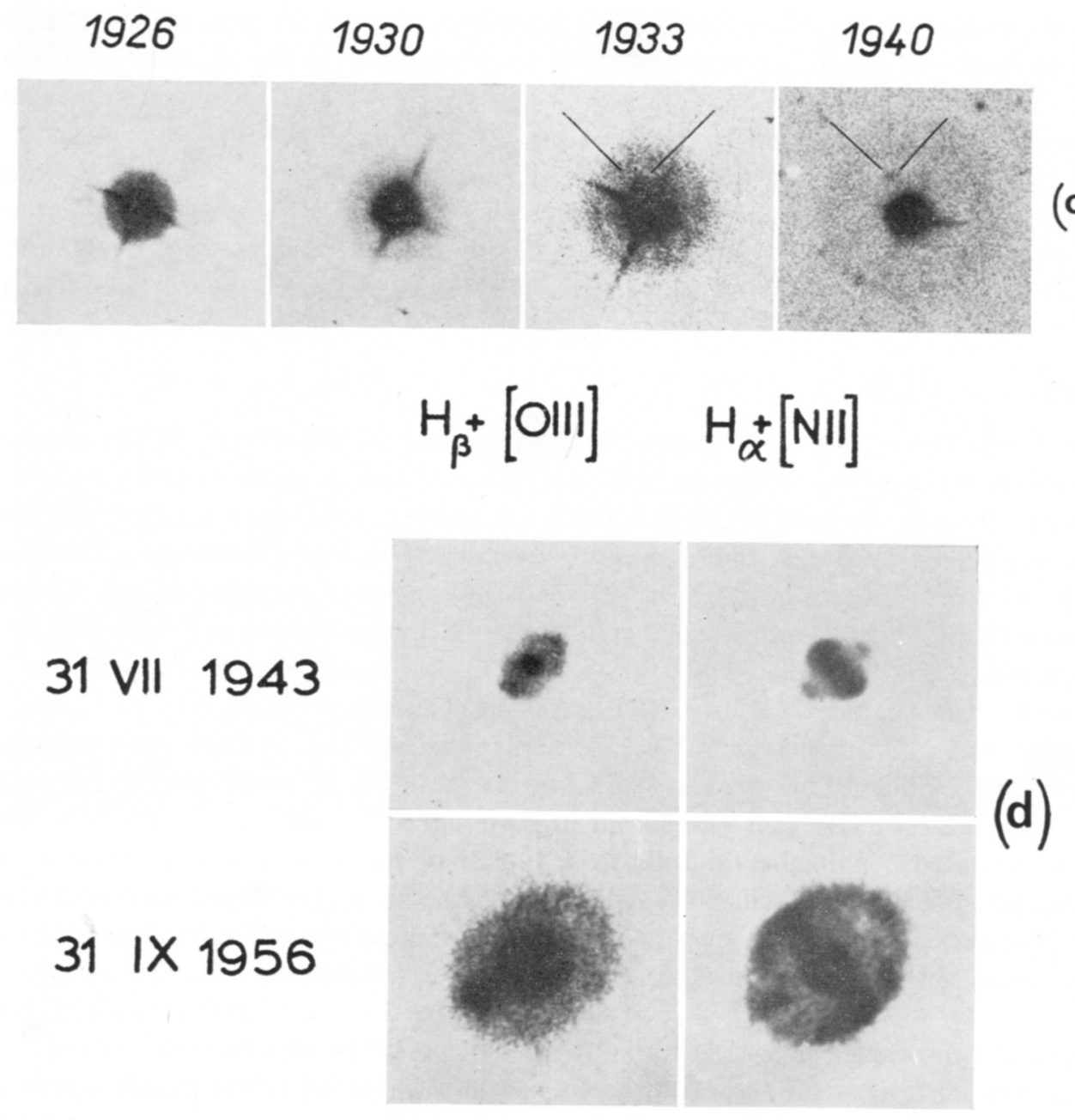

Fig. 1. Several novae: (a) N Cyg 1920; (b) N Aur 1891; (c) N Aql 1918; (d) N Her 1934.

loss. Most novae are binary systems in which both components are dwarf stars. Suddenly one of the stars increases in brightness, with an amplitude of the flare up to $12^{m}$ (a factor of 100000 ). Several years after the outburst a small envelope appears around the nova. Some examples of nova envelopes are shown in Figure 1, in which all pictures were taken at Mount Wilson and Palomar Observatories (Mustel' and Boyarchuk, 1970). The expansion velocities are rather large: about $2000 \mathrm{~km} \mathrm{sec}^{-1}$ for fast novae and about $300 \mathrm{~km} \mathrm{sec}^{-1}$ for slow ones. In all cases the expansion velocities are much larger than the velocities of escape, which are equal to a few $\mathrm{km} \mathrm{sec}^{-1}$. Such large velocities of expansion imply that the envelopes of novae can last for only a short time (several tens of years after the outburst). As a result, the mass of the envelope has been determined for only very few novae, namely for those with the outburst 
TABLE I

Mass loss from novae

\begin{tabular}{lccc}
\hline Name & $\begin{array}{l}V_{\text {exp }} \\
\left(\mathrm{km} \mathrm{sec}^{-1}\right)\end{array}$ & Mass of shell $\left(M_{\odot}\right)$ & References \\
\hline & & & \\
N Lac 1936 & 2200 & $3.6 \times 10^{-5}$ & 1 \\
N Aq1 1918 & 1700 & $1.4 \times 10^{-4}$ & 1 \\
N Per 1901 & 1340 & $3.8 \times 10^{-5}$ & 1 \\
N Pic 1925 & 800 & $10^{-4}$ & 1 \\
N Her 1934 & 290 & $4.6 \times 10^{-5}$ & 2 \\
Recurrent Nova & & & \\
RS Oph 1933 & & $5 \times 10^{-6}$ & 3 \\
1958 & 1000 & $2 \times 10^{-7}$ & 4
\end{tabular}

(1) Pottasch (1959); (2) Mustel' and Boyarchuk (1970); (3) Sayer (1937); (4) Folkart et al. (1964).

in the last century. Table I shows the expansion velocities and the masses of the envelope for several novae. The average mass equals $7 \times 10^{-5} M_{\odot}$. According to Kukarkin (1954) and Allen (1955) about 100 novae explode in the Galaxy per year, so that novae put back into the interstellar medium a mass of about $7 \times 10^{-3} M_{\odot} \mathrm{yr}^{-1}$.

It appears that a nova may lose mass for a long time after the dramatic explosion. For example, recently Nather and Warner (1969) have found that the period of the binary system DQ Her ( = N Her 1934) increases with time. This means that DQ Her continues to lose mass 35 years after the outburst. The value of the period change corresponds to a mass loss of $1.1 \times 10^{-7} M_{\odot} \mathrm{yr}^{-1}$. One can assume that other old novae also have mass loss still going on at about the same rate, because all of them show emission lines in their spectra indicating gaseous streams. In order to estimate a total rate of mass loss for all novae after the outburst we need to know how long old novae continue their activity. DQ Her had its outburst in 1934; T Aur had its outburst in 1891, and it still seems active. There are many objects in the Galaxy which have spectral and photometric characteristics similar to that of old novae. Therefore, one can assume that the activity of old novae continues at least $10^{2} \mathrm{yr}$. If we assume that each old nova has the same rate of mass loss as that of DQ Her then the total rate will be more than $1.1 \times 10^{-3} M_{\odot} \mathrm{yr}^{-1}$, less than the rate of mass loss during the outbursts.

Finally consider the 'recurrent novae', a small group of novae, for which more than one outburst has been observed. Only for one recurrent nova - RS Oph - has the rate of mass loss during the outburst been determined. Table I shows the expansion velocities and the mass of the envelope. One can see that in this case the mass of the ejected material is a hundred times less than that of the ordinary novae. The total number of recurrent novae in the Galaxy is at least ten times less than that of the novae. We can conclude that the mass ejected by all recurrent novae during many outbursts is probably less than that ejected by all novae during single outbursts. 


\section{Symbiotic Stars}

The term 'symbiotic stars' is widely used for objects whose spectra represent a combination of absorption features of low temperature stars (TiO bands; CaI, CaII absorption lines, etc.) with emission lines of high excitation ([FevII], [Nev], etc.). The brightness of these stars varies with amplitudes up to $3^{m}$ and with a period of several years. The current hypothesis states that the object is a binary. One of the components is a late-type giant and the other one is a hot, small star which is the source of excitation of a nebula surrounding both components. One can assume that the nebula has been ejected from one of the two stars. Unfortunately the angular sizes of the nebulae are much less than $1^{\prime \prime}$ and nobody has seen them on direct pictures. Several astronomers (Merrill, 1959; Boyarchuk, 1967) have measured the widths of emission lines, which characterize the expansion velocity of the nebula. Table II shows the expansion velocities and the masses of nebulae for some symbiotic stars. The total mass of both stars is about $10 M_{\odot}$, the radius of the nebula is about $10^{3} R_{\odot}$ (Boyarchuk, 1969) and so the escape velocity is about $60 \mathrm{~km} \mathrm{sec}^{-1}$, which is less than the observed expansion velocities for most of the stars. From these data it follows that the average mass in the nebula of a symbiotic star is about $5 \times 10^{-4} M_{\odot}$. Unfortunately we do not know the lifetime of symbiotic stars. If we assume that the lifetime of symbiotic stars is close to

TABLE II

Mass loss of symbiotic stars

\begin{tabular}{lcc}
\hline Name & $V_{\exp }\left(\mathrm{km} \mathrm{sec}^{-1}\right)$ & Mass of shell $\left(M_{\odot}\right)$ \\
\hline AG Dra & & $1.5 \times 10^{-4}$ \\
Z And & 70 & $10^{-4}$ \\
AG Peg & $100-400$ & $10^{-4}$ \\
AX Per & & $4 \times 10^{-4}$ \\
CI Cyg & & $<5 \times 10^{-5}$ \\
BF Cyg & & $4.2 \times 10^{-3}$ \\
\hline
\end{tabular}

that of planetary nebulae then the rate of mass loss is about $5 \times 10^{-8} M_{\odot} \mathrm{yr}^{-1}$. The total number of symbiotic stars in the Galaxy is about $10^{3}$ (Boyarchuk, 1970a) and the total rate of mass loss of symbiotic stars is about $5 \times 10^{-5} M_{\odot} \mathrm{yr}^{-1}$.

One can obtain some information about the mass loss in large flares. It is known that during a large flare the spectra of symbiotic stars show many lines with P Cygnitype profiles (Swings and Struve, 1940; Gauzit, 1955). The relative displacement of absorption and emission components corresponds to an expansion velocity of about $120 \mathrm{~km} \mathrm{sec}^{-1}$. Boyarchuk (1970b) studied the large flare of $Z$ And of 1961. The hot component had a radius of $70 R_{\odot}$. If the mass equals $1 M_{\odot}$, the escape velocity is 80 $\mathrm{km} \mathrm{sec}^{-1}$, less than the observed velocity. The density of the region where the absorption lines were formed was found to be $5 \times 10^{-14} \mathrm{~g} \mathrm{~cm}^{-3}$ and the continuous loss rate $2 \times 10^{-6} M_{\odot} \mathrm{yr}^{-1}$. Since the $\mathrm{P}$ Cygni-type profile has been observed during two or 
three months and the frequency of flares is approximately one per two years, the rate of mass loss is about $10^{-7} M_{\odot} \mathrm{yr}^{-1}$. The total mass loss from all symbiotic stars is about $10^{-4} M_{\odot} \mathrm{yr}^{-1}$.

\section{T Tauri Stars}

$\mathrm{T}$ Tauri-type stars are characterized by irregular light variations which may reach a few magnitudes and by the presence of emission lines, especially fluorescent lines of FeI. The stars have spectral classes $G$ through $M$ and they are located in the mainsequence region and in the subgiant region of the H-R diagram. The majority of these stars are evidently connected with dark and with bright diffuse nebulae. There is considerable evidence that $\mathrm{T}$ Tauri stars are very young and still in the process of

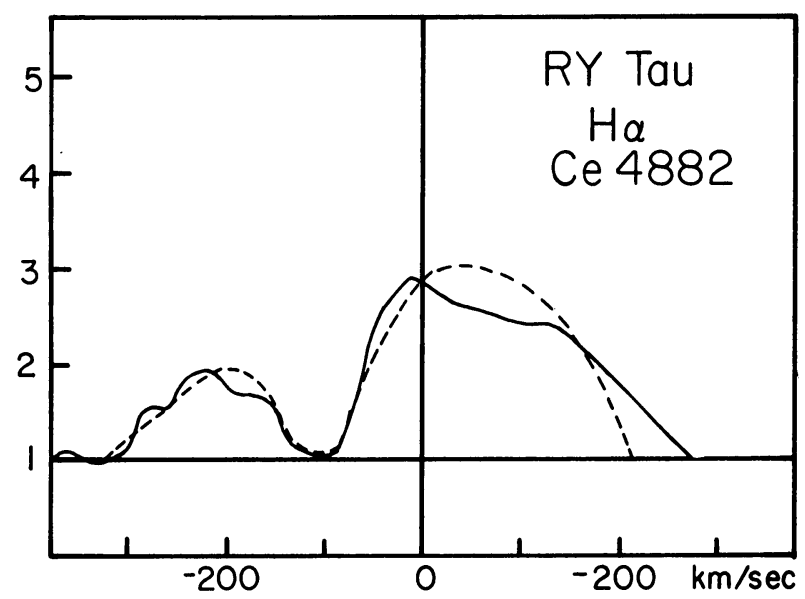

Fig. 2. Comparison of the observed profile of $\mathrm{H} \alpha$ (dashed line) and the predicted profile (solid line) in the spectrum of RY Tau (Kuhi, 1964).

gravitational contraction. The strongest emission lines - $\mathrm{HI}$ and $\mathrm{Ca} I \mathrm{I}(\mathrm{H}$ and $\mathrm{K})-$ usually have P Cygni-type profiles: emission components 5 to $6 \AA$ wide which have violet-displaced absorption features. Such profiles indicate the presence of material moving away from the star. As there is no sign of the returning of material, we can conclude that real mass loss takes place. Several determinations of the mass loss rate have been made. From a rough investigation of spectrograms of the very luminous T Tau star $\mathrm{LK} \mathrm{H}_{\alpha} 120$ Herbig (1962) estimated a mass loss rate of about $10^{-5} M_{\odot} \mathrm{yr}^{-1}$. Varsavsky (1962) used the density determined by Osterbrock (1958) from the relative intensities of the [OII] $\lambda \lambda 3727,3729$ doublet, to obtain the result that the mass loss rate from $\mathrm{T}$ Tau itself is about $10^{-7} M_{\odot} \mathrm{yr}^{-1}$. The most detailed analysis of the mass loss rate has been carried out by Kuhi (1964). He assumed that the stars have a spherically symmetric envelope moving radially outward and subjected only to the deceleration of gravitational forces. The inner part of the envelope provides the emission lines while the outer part of an envelope provides the absorp- 
tion feature. Kuhi calculated a set of model profiles and by fitting these to the observed profile he determined the density at the stellar surface and the expansion velocity (see Figure 2). Then he obtained the mass-loss rates. The results for six $\mathrm{T}$ Tauri stars are shown in Table III. The weighted mean rate is $3.7 \times 10^{-8} M_{\odot} \mathrm{yr}^{-1}$.

TABLE III

Mass-loss rates of T Tauri stars (after Kuhi, 1966)

\begin{tabular}{lll}
\hline Star & $V_{\exp }\left(\mathrm{km} \mathrm{sec}^{-1}\right)$ & $\mathrm{d} M / \mathrm{d} t\left(M_{\odot} \mathrm{yr}^{-1}\right)$ \\
\hline RU Lup & 200 & $1.4 \times 10^{-7}$ \\
Lk H ${ }_{\alpha} 120$ & 180 & $5.9 \times 10^{-7}$ \\
AS 209 & $\sim 0$ & $0.65 \times 10^{-7}$ \\
GW Ori & 80 & $0.35 \times 10^{-7}$ \\
T Tau & 140 & $0.35 \times 10^{-7}$ \\
RY Tau & 100 & $0.31 \times 10^{-7}$
\end{tabular}

If we assume that the total number of $T$ Tauri-type stars in the Galaxy is $10^{6}$ (Kuhi, 1966) they are returning about $0.04 M_{\odot} \mathrm{yr}^{-1}$ to the interstellar medium.

\section{U Geminorum Stars}

The main characteristic of $U$ Geminorum stars is that the brightness increases now and then by several magnitudes, but after one to two days it returns to its former value. It has been established that $U$ Geminorum stars are binary systems, both components of which are dwarf stars. It is unknown which component has an outburst. Nobody has yet observed nebulosities around the stars. Grant and Abt (1959) and Zuckermann (1962) have found that the color of SS Cyg is affected by reddening. They decided that the reddening is due to the absorption in a circumstellar envelope which was formed by the ejected material. Some doubt about their conclusion arises from the uncertainty of the intrinsic color of SS Cyg. All this leads to difficulty in the determination of the mass-loss rate. Walker and Chincarini (1968) have investigated the radial velocity variation of SS Cyg and have found that the length of the period increases with time. The change of the period corresponds to a mass-loss rate of $1.8 \times 10^{-7} M_{\odot} \mathrm{yr}^{-1}$. The total number of U Geminorum stars in the Galaxy is $10^{7}$ (Gorbatskii 1970). If we suppose that SS Cyg is typical, then the total mass loss rate from U Geminorum stars will be about $2 M_{\odot} \mathrm{yr}^{-1}$.

\section{UV Ceti Stars}

The UV Ceti variables are dwarf stars of spectral classes dM3e - dM6e. They are characterized by rare and very short (several minutes) flares with amplitudes up to $6^{m}$. So far no nebulae have been seen around these stars. The faintness of UV Ceti stars and the short duration of the flares does not permit as yet the careful investigation of the line profiles. The nature of flares is still unknown. The best hypothesis 
is the assumption that the observed flares arise in chromospheres and coronae surrounding UV Ceti stars. On the basis of this hypothesis Kahn (1969), using the observations by Lovell (1969) and by Kunkel (1969), has found that the star YZ CMi loses $3 \times 10^{-12} M_{\odot} \mathrm{yr}^{-1}$. Gershberg (1968) has evaluated an upper limit to the mass loss from an analysis of the light changes during the flares. He has found that the rate of the mass loss is less than $10^{-11} M_{\odot} \mathrm{yr}^{-1}$. According to Gershberg (1968) the Galaxy contains $10^{9}$ UV Ceti stars. Then the total loss rate will be about $10^{-2} M_{\odot} \mathrm{yr}^{-1}$.

\section{Summary}

Table IV is a summary. The first column gives the type of variable stars, the second the mass loss rate per star, the third the number $n$ of such stars in the Galaxy, the fourth the total mass loss rate of all stars of the type considered, the fifth the ejection velocities.

TABLE IV

Summary of mass-loss rates

\begin{tabular}{|c|c|c|c|c|}
\hline Name & $\mathrm{d} M / \mathrm{d} t\left(M_{\odot} \mathrm{yr}^{-1}\right)$ & $n$ & $n(\mathrm{~d} M / \mathrm{d} t)\left(M_{\odot} \mathrm{yr}^{-1}\right)$ & $V_{\exp }\left(\mathrm{km} \mathrm{sec}^{-1}\right)$ \\
\hline \multicolumn{5}{|l|}{ Novae } \\
\hline outburst & & $10^{2}$ & $7 \times 10^{-3}$ & 1000 \\
\hline cont. eject. & $1.1 \times 10^{-7}$ & $10^{4}$ & $10^{-3}$ & \\
\hline Symbiotic stars & $5 \times 10^{-8}$ & $10^{3}$ & $5 \times 10^{-5}$ & 100 \\
\hline T Tau stars & $3.7 \times 10^{-8}$ & $10^{6}$ & $4 \times 10^{-2}$ & 100 \\
\hline U Gem stars & $1.8 \times 10^{-7}$ & $10^{7}$ & 1.8 & \\
\hline UV Cet stars & $10^{-11}$ & $10^{9}$ & $10^{-2}$ & \\
\hline
\end{tabular}

It would follow from Table 4 that $\mathrm{U}$ Geminorum stars are the main contributor of the material into space. However this conclusion is not convincing because the value of the mass loss rate of $U$ Geminorum stars is based on only one star and the total number of these stars in the Galaxy may be in error by a factor of 10 . The last statement may be true for other kinds of eruptive stars as well.

\section{References}

Allen, C. W.: 1955, Monthly Notices Roy. Astron. Soc. 114, 387.

Boyarchuk, A. A.: 1967, Astron. Zh. 44, 12 (1967, Soviet Astron. 11, 8).

Boyarchuk, A. A.: 1969, in Proc. Fourth IAU Colloquium on Variable Stars, Budapest, p. 395.

Boyarchuk, A. A.: 1970a, in Eruptive Stars, Nauka, Moscow, p. 113.

Boyarchuk, A. A.: 1970b, Astrofiz., in press.

Folkart, W., Pecker, J.-C., and Pottasch, S. R.: 1964, Ann. Astrophys. 27, 252.

Gauzit, J.: 1955, Ann. Astrophys. 18, 354.

Gershberg, R. E.: 1968, in Problems of the Stellar Evolution and Variable Stars, Nauka, Moscow, p. 50.

Gorbatskii, V. G.: 1970, in Eruptive Stars, Moscow, in press.

Grant, G. and Abt, H. A.: 1959, Astrophys. J. 129, 323.

Herbig, G. H.: 1962, in Symposium on Stellar Evolution (ed. by J. Sahade), Observatorio Astronómico, La Plata, Argentina, p. 23. 
Huang, S. S.: 1956, Astron. J. 61, 49.

Kahn, F. D.: 1969, Nature 222, 1130.

Kuhi, L. V.: 1964, Astrophys. J. 140, 1409.

Kuhi, L. V.: 1966, in Stellar Evolution (ed. by R. F. Stein and A. G. W. Cameron), Plenum Press. New York, p. 373.

Kukarkin, B. V.: 1954, Variable Stars and Stellar Evolution, Moscow.

Kunkel, W. E.: 1969, Nature 222, 1129.

Lovell, B.: 1969, Nature 222, 1127.

Merrill, P. W.: 1959, Astrophys. J. 129, 44.

Mustel', E. R. and Boyarchuk, A. A.: 1970, Astrophys. Space Sci. 6, 183.

Nather, R. E. and Warner, B.: 1969, Monthly Notices Roy. Astron. Soc. 143, 145.

Osterbrock, D. E.: 1958, Publ. Astron. Soc. Pacific 70, 399.

Pottasch, S. R.: 1959, Ann. Astrophys. 22, 394.

Sayer, A. R.: 1937, Ann. Harv. Coll. Observ. 105, 21.

Swings, P. and Struve, O.: 1940, Astrophys. J. 91, 546.

Varsavsky, C. M.: 1962, in Symposium on Stellar Evolution (ed. by J. Sahade), Observatorio Astronómico, La Plata, Argentina, p. 33.

Walker, M. F. and Chincarini, G.: 1968, Astrophys. J. 154, 157.

Zuckermann, M.-C.: 1962, Ann. Astrophys. 24, 431. 